\title{
On the impact of non-Gaussian wind statistics on wind turbines - an experimental approach
}

\author{
Jannik Schottler ${ }^{1}$, Nico Reinke ${ }^{1}$, Agnieszka Hölling ${ }^{1}$, Jonathan Whale ${ }^{2}$, Joachim Peinke ${ }^{1}$, and \\ Michael Hölling ${ }^{1}$ \\ ${ }^{1}$ ForWind, University of Oldenburg, Institute of Physics, Küpkersweg 70, 26129 Oldenburg, Germany \\ ${ }^{2}$ Murdoch University, School of Engineering and Information Technology, Murdoch, WA, 6150, Australia \\ Correspondence to: Jannik Schottler (jannik.schottler@uni-oldenburg.de)
}

Received: 11 July 2016 - Published in Wind Energ. Sci. Discuss.: 13 July 2016

Revised: 18 November 2016 - Accepted: 4 December 2016 - Published: 10 January 2017

\begin{abstract}
The effect of intermittent and Gaussian inflow conditions on wind energy converters is studied experimentally. Two different flow situations were created in a wind tunnel using an active grid. Both flows exhibit nearly equal mean velocity values and turbulence intensities but strongly differ in their two point statistics, namely their distribution of velocity increments on a variety of timescales, one being Gaussian distributed, and the other one being strongly intermittent. A horizontal axis model wind turbine is exposed to both flows, isolating the effect on the turbine of the differences not captured by mean values and turbulence intensities. Thrust, torque and power data were recorded and analyzed, showing that the model turbine does not smooth out intermittency. Intermittent inflow is converted to similarly intermittent turbine data on all scales considered, reaching down to sub-rotor scales in space. This indicates that it is not correct to assume a smoothing of intermittent wind speed increments below the size of the rotor.
\end{abstract}

\section{Introduction}

Wind energy converters (WECs) work in a turbulent environment and are therefore turbulence-driven systems. The turbulent wind interacts with the system dynamics, resulting in the output parameters of a wind energy converter system such as power, mechanical loads or other quantities of interest.

Generally, the characteristics of the output dynamics of a WEC need to be understood in detail for multiple reasons. Power fluctuations have been reported in numerous studies, causing challenges in grid stability (e.g., Chen and Spooner, 2001; Carrasco et al., 2006; Sørensen et al., 2007). Drivetrain and gearbox failure rates remain high, adding to the cost of energy since gearboxes are among the most expensive parts of WECs. These types of failures are likely to be linked to torque fluctuations (e.g., Musial et al., 2007; Feng et al., 2013). Next, turbulent wind affects extreme and fatigue loads, which is clearly related to the lifetime of WECs (Burton et al., 2001).

Wind dynamics in the atmospheric boundary layer have been investigated extensively. Here, one has to differentiate between analyses concerning the statistics of the wind speed values and velocity increments. The wind velocities might become anomalously distributed due to large-scale meteorological events like downbursts or thunderstorms (De Gaetano et al., 2014). Velocity increments, on the other hand, statistically characterize the temporal aspect of fluctuations, whose non-Gaussian statistics are well-known from small-scale turbulence (Frisch, 1995). Active systems, like wind turbines discussed here, adapt to actual wind situations. Thus, in this paper we focus on wind speed changes within seconds, i.e., by the corresponding increments. Numerous studies have reported on non-Gaussian characteristics of wind speed increments; see, e.g., Boettcher et al. (2003), Liu et al. (2010), Morales et al. (2012), and Wächter et al. (2012). Furthermore, findings of non-Gaussian wind statistics have been implemented in simulations by a variety of methods; see, e.g., Nielsen et al. (2007), Mücke et al. (2011), and Gong and Chen (2014).

In the field of wind energy research, it is still unclear to what extent wind dynamics transfer to the parameters of a WEC such as loads, power etc. This most likely depends on 
the relevant timescales, which change with the system dynamics. Therewith, the conversion from wind to power, loads etc. vary with the turbine type. Consequently, it is important what scales in time and space are relevant to quantify the impact of turbulent wind on WECs (van Kuik et al., 2016), and scale-dependent analyses become necessary.

Mücke et al. (2011) found that intermittent inflow conditions do not significantly affect rain flow distributions of the torque. However, similarly intermittent torque increments based on a numeric wind turbine model used in the aeroelastic tool FAST (Jonkman and Buhl Jr., 2005) in combination with AeroDyn (Moriarty and Hansen, 2005) were found. Gong and Chen (2014) investigated the short- and long-term extreme response distributions of a wind turbine during Gaussian and non-Gaussian inflow conditions using FAST. The extreme turbine responses to non-Gaussian inflow were considerably larger than the responses to Gaussian wind. However, Berg et al. (2016) recently reported a vanishing effect of non-Gaussian turbulence on extreme and fatigue loads based on wind fields generated by largeeddy simulation (LES) in combination with aeroelastic load simulations using HAWC2 (Larsen and Hansen, 2007). It was concluded that non-Gaussianity in sub-rotor-sized eddies is filtered by the rotor. Using field data, Milan et al. (2013) showed that multi-MW WECs convert intermittent wind speeds to turbulent-like intermittent power with fluctuations down to the scale of seconds. Even on the scale of an entire wind farm, intermittent power output was reported. To summarize, different simulations and data from real turbines deliver an inconclusive answer to our question about the conversion from turbulent inflow to wind turbine data. It is not clear to what extent non-Gaussian flow conditions transfer to turbine parameters. At the same time, this is a very important aspect in the design process of wind turbines and in the wind field models used. Wrong assumptions about the conversion from turbulence characteristics to turbine data might lead to faulty dimensions and problems in the integration of wind energy in the power grid.

Using wind tunnel experiments, we contribute to the ongoing discussion on the conversion process of non-Gaussian wind statistics to wind turbine data such as power, thrust and torque. A model wind turbine and an active grid for flow manipulation were used in order to examine to what extent Gaussian-distributed and highly intermittent wind speeds affect the model turbine dynamics differently.

This paper is organized as follows: Sect. 2 gives an overview of commonly used methods for characterizing wind speed time series, parts of which are applied to offshore measurement data and simulated wind speed time series. Mathematical tools used throughout this paper are introduced here. Next, Sect. 3 describes the experimental methods used, including the setup, the definition of examined quantities and their processing. Section 4 shows the results of the experiments, which are discussed in Sect. 5. Finally, Sect. 6 gives the conclusion of the findings.

\section{Atmospheric flows}

Since WECs work in turbulent wind conditions, a proper characterization of these conditions becomes necessary (van Kuik et al., 2016). The industry standard IEC 61400-1 defines procedures for wind field description (International Electrotechnical Commission, 2005). Power spectral densities, along with 10 min mean values and turbulence intensities, are considered. Therewith, only the first two statistical moments of a velocity time series are taken into account.

In this section, we give a brief overview of the methods used in the industry standard and beyond, along with their mathematical background, without claims of completeness. Furthermore, the methods of data analysis used in this study are introduced. We refer to Morales et al. (2012) for a more detailed elaboration.

A general first step in characterizing a time series of wind velocities, $u(t)$, is the definition of velocity fluctuations (Burton et al., 2001),

$u^{\prime}(t)=u(t)-\langle u\rangle$

where $\langle u\rangle$ denotes the mean value of $u(t)$. A commonly used quantification of the general level of turbulence is the turbulence intensity $(\mathrm{TI})$,

$\mathrm{TI}=\frac{\sigma_{\widetilde{T}}}{\langle u\rangle_{\widetilde{T}}}$,

with $\sigma_{\widetilde{T}}$ being the standard deviation of $u(t)$ during the time $\widetilde{T}$ (Burton et al., 2001). Accordingly, $\langle u\rangle_{\widetilde{T}}$ denotes the mean value over the same time span, which is typically $10 \mathrm{~min}$ in industry standards. Notice, since $\sqrt{\left\langle u^{\prime 2}(t)\right\rangle_{\widetilde{T}}}=\sigma_{\widetilde{T}}$, only the first two statistical moments of the one point quantity $u^{\prime}$ are considered when describing a velocity time series by its fluctuations and/or turbulence intensity as previously defined.

Going one step further in the sense of two point quantities, we will consider velocity changes during a time lag $\tau$ and refer to them as velocity increments,

$u_{\tau}(t)=u(t+\tau)-u(t)$

throughout this paper. It is important to distinguish between a statistical description of the fluctuations and the increments. In stationary turbulence, $u^{\prime}(t)$ is close to a Gaussian distribution, whereas increment statistics increasingly deviate from Gaussianity (Frisch, 1995), which is also shown by Morales et al. (2012) for offshore data. The $n$ th-order moments of $u_{\tau}(t)$ are commonly referred to as $n$ th-order structure functions (Wächter et al., 2012). The second-order structure function

$\left\langle u_{\tau}(t)^{2}\right\rangle=\left\langle(u(t+\tau)-u(t))^{2}\right\rangle$ 
is directly linked to the autocorrelation function $R_{u u}(\tau)$,

$$
\begin{aligned}
\left\langle u_{\tau}(t)^{2}\right\rangle & =2\left\langle u(t)^{2}\right\rangle-2\langle u(t) u(t+\tau)\rangle \\
& =2\left\langle u(t)^{2}\right\rangle\left(1-R_{u u}(\tau)\right),
\end{aligned}
$$

with the assumption that $\left\langle u(t)^{2}\right\rangle=\left\langle u(t+\tau)^{2}\right\rangle$. The autocorrelation function

$R_{u u}(\tau)=\frac{1}{\sigma_{u}^{2}}\langle u(t) u(t+\tau)\rangle$

is connected to the power spectral density (PSD) by the Fourier transformation. ${ }^{1}$ Therewith, the PSD, which is used broadly in wind field models such as the well-known Kaimal model (Kaimal et al., 1972), comprises the same information as the second-order structure function.

In order to include all higher-order structure functions, $\left\langle u_{\tau}^{n}\right\rangle$, we will consider the probability density functions (PDFs) of velocity increments, $p\left(u_{\tau}\right)$, for different time lags $\tau$ and refer to them as increment $P D F$. We normalize $u_{\tau}$ by its standard deviation,

$\sigma_{\tau}=\sqrt{\frac{1}{N-1} \sum_{i=1}^{N}\left(u_{\tau_{i}}-\left\langle u_{\tau}\right\rangle\right)^{2}}$,

for better visual comparison. The statistical error of each bin of the PDF is estimated by $1 / \sqrt{\tilde{n}}$, where $\tilde{n}$ is the number of events in the respective bin. Throughout our analyses, values with a statistical error exceeding $10 \%$ are marked with a red $x$.

For design load calculations, different turbulence models are used. One, which is suggested by the IEC standard, is the Kaimal model, which considers power spectral densities and features merely Gaussian statistics. In this paper, we investigate to what extent wind characteristics not captured by standard models impact wind turbines. Furthermore, we consider a synthetic wind speed time series based on the Kaimal turbulence model, created using the software TurbSim (Jonkman, 2009), and compare it to offshore wind speed measurements taken from the FINO1 offshore measurement platform at $80 \mathrm{~m}$ height. The offshore data set is documented by Westerhellweg et al. (2012). We considered $10 \mathrm{~Hz}$ data for 1 year, and 10 min records of $7 \mathrm{~m} \mathrm{~s}^{-1} \leq\langle u(t)\rangle_{10 \mathrm{~min}} \leq 8 \mathrm{~m} \mathrm{~s}^{-1}$ were selected. The approximately 3700 records were then combined and used in this analysis in order to ensure close-tostationary conditions. It was shown by Morales et al. (2012) that such a constraint filters out intermittency effects caused by nonstationary conditions on large scales and thus enables us to more properly study small-scale turbulence effects. It should be noted that only the mean value of one 10 min block is within $7.5 \pm 0.5 \mathrm{~m} \mathrm{~s}^{-1}$. During this time span, samples outside of this interval are included. Table 1 shows the mean

\footnotetext{
${ }^{1} \mathcal{F}\left\{R_{u u}(\tau)\right\}=S(f)$, with $\sigma_{u}^{2}=\int S(f) \mathrm{d} f$ and $S(f)$ being the power spectral density (Press et al., 1992).
}

Table 1. First two statistical moments and turbulence intensities of a synthetic wind speed time series based on the Kaimal model and offshore data (FINO1). Values are rounded to two decimal places.

\begin{tabular}{lrrr}
\hline Time series & $\langle u\rangle\left[\mathrm{m} \mathrm{s}^{-1}\right]$ & $\sigma_{u}\left[\mathrm{~m} \mathrm{~s}^{-1}\right]$ & TI [\%] \\
\hline Kaimal & 7.51 & 0.54 & 7.21 \\
FINO1 & 7.50 & 0.54 & 7.18 \\
\hline
\end{tabular}

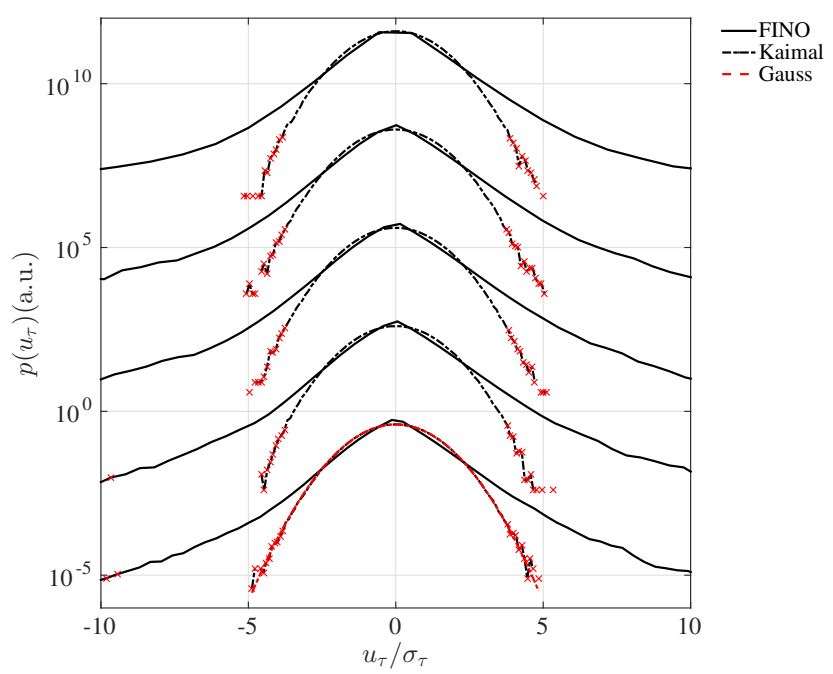

Figure 1. $p\left(u_{\tau}\right)$ for data sets based on the Kaimal model (dashed black line) and for offshore measurements, conditioned so that $\langle u\rangle=7.5 \pm 0.5 \mathrm{~m} \mathrm{~s}^{-1}$ (solid black). The PDFs for each scale are shifted vertically for better comparison, which is done throughout this paper. Scales from top to bottom $\tau=\{1,5,10,30,60 \mathrm{~s}\}$.

values, standard deviations and turbulence intensities of both data sets. As can be seen, the synthetic time series and the field measurements are very similar regarding their mean values and turbulence intensities (see Table 1). Going further, Fig. 1 shows $p\left(u_{\tau}\right)$ of both data sets, showing distinct differences regarding their distributions of increments. The Kaimal model comprises purely Gaussian statistics, while the offshore data features intermittent increment distributions. As shown in Fig. 1, certain characteristics of a wind speed time series, in particular extreme velocity increments, are not reflected correctly using standard methods. In this paper, we elaborate if and to what extent flow characteristics that are not captured by the standards (e.g., the first two statistical moments) impact wind turbines. We follow an experimental approach using a model wind turbine in a wind tunnel equipped with an active grid, allowing the generation of various turbulent inflow conditions. By tuning the intermittency while preserving mean wind speeds and turbulence intensities, the effect of intermittency is isolated. 


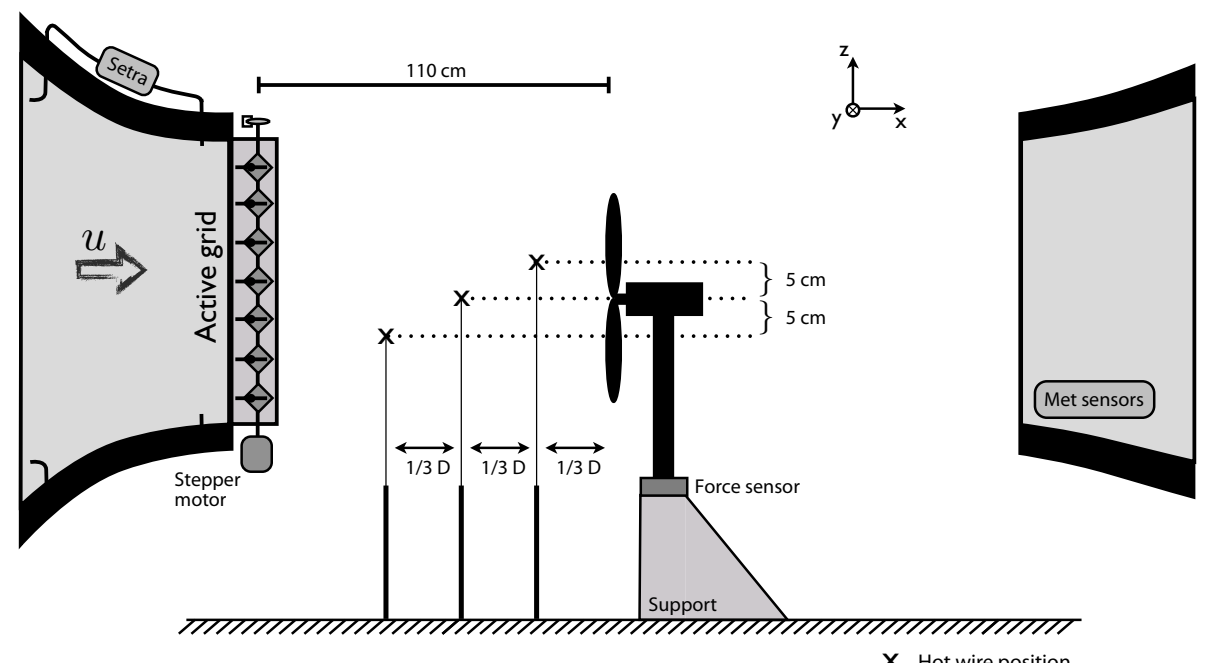

Figure 2. Schematic drawing of the experimental setup, side view. Scales do not match, $D=0.58 \mathrm{~m}$.

\section{Methods}

\subsection{Experimental setup}

\subsubsection{Wind tunnel and active grid}

The experiments were conducted in a wind tunnel of the University of Oldenburg in open jet configuration. The outlet of $0.8 \mathrm{~m} \times 1 \mathrm{~m}$ (height $\times$ width) was equipped with an active grid for turbulence generation with a similar design as described by Weitemeyer et al. (2013). The grid is made of nine vertical and seven horizontal axes with square metal plates attached. To allow an individual motion of the axes and thus flow manipulation, 16 stepper motors were used. However, throughout the experiments, all axes were excited simultaneously. We define a flap angle $\alpha$, whereas $\alpha=0^{\circ}$ is in alignment with the main flow direction (open) and $\pm 90^{\circ}$ corresponds to maximum blockage. At $\alpha=0^{\circ}$, the blockage of the grid is approximately $6 \%$, considering the crosssectional area of the grid in relation to the wind tunnel outlet.

The excitation protocols of the motors were designed so that two different flow situations with the same mean wind velocities and comparable turbulence intensities were realized. At the same time, they strongly differ in their distributions of increments: one flow (A) being Gaussian distributed, the other one (B) being highly intermittent on a broad range of timescales, which shows a distinctly heavy-tailed distribution of velocity increments. The resulting time series are discussed in Sect. 4.1. The excitation protocol resulting in the intermittent flow featured an active flow modulation, where $\alpha$ was changed appropriately at a maximal rate of $50 \mathrm{~Hz}$. For the Gaussian flow, the axes were not moved dynamically so that $\dot{\alpha}=0^{\circ}$.

The flows considered were characterized using three single-wire hot-wire probes simultaneously in one plane normal to the main flow direction. The probes were arranged so that one was located at the position of the model wind turbine's hub and the other two in $0.6 \mathrm{D}$ distance displaced in $y$ and $z$ directions (see Fig. 2). It should be noted that the turbine was not installed during flow characterization. The hot wires are $1.25 \mathrm{~mm}$ long with a diameter of $5 \mu \mathrm{m}$. A constant-temperature anemometry (CTA) module (Dantec 9054N0802) with a built-in low-pass filter set to $5 \mathrm{kHz}$ was used. Data were recorded at $10 \mathrm{kHz}$ for $25 \mathrm{~min}$ using a National Instruments cRIO-9074 real-time controller with inhouse built LabVIEW software. When analyzing the flows, spatially averaged mean values of the three simultaneous measurements,

$u(t)=\frac{1}{3} \sum_{i=1}^{3} u_{i}(t)$,

are considered, where the index $i$ denotes the respective hot wire. Following the concept of a rotor-effective wind speed as used by Schlipf et al. (2013), this approach is more appropriate for describing the wind speed affecting the rotor than a single-point measurement. It should be noted that our results are hardly affected by using averaged measurements as opposed to data of the central hot wire. The distance from the active grid to the rotor and hence the hot wires was $1.1 \mathrm{~m}$, which was set as a compromise between two aspects: first, the further away from the outlet, the greater the influence of the emerging shear layer (Mathieu and Scott, 2000), which should be limited. Second, the interaction of the rotor's blockage with the active grid increases with shorter distances. The evolution of the turbulence intensity and intermittency was also found to decay constantly around $1 \mathrm{~m}$ behind the grid (Weitemeyer et al., 2013). Consequently, a distance of $1.1 \mathrm{~m}$ was chosen to balance the effects described. 


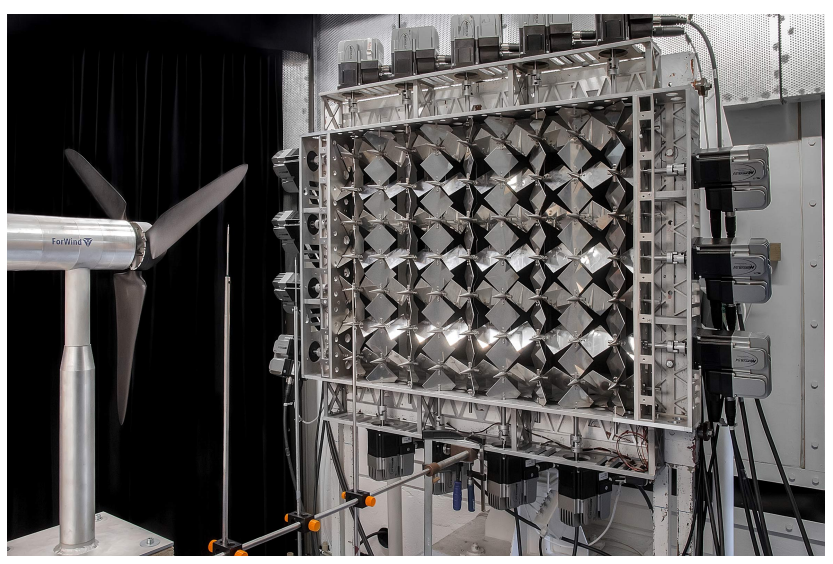

Figure 3. The model wind turbine and the active grid installed in a wind tunnel of the University of Oldenburg.

\subsubsection{Model wind turbine}

A three-bladed horizontal-axis model wind turbine with a rotor diameter of $D=0.58 \mathrm{~m}$ was used. The vacuum-casted rotor blades are based on a SD7003 airfoil profile. The turbine is dynamically controlled with an operating tip speed ration (TSR) comparable to modern full-scale turbines. Further details on the turbine design are described by Schottler et al. (2016). For details about the blade design, see Odemark (2012). We consider the electrical power

$P=P_{\mathrm{el}}=U_{\mathrm{gen}} \cdot I$,

where $U_{\text {gen }}$ is the generator voltage and $I$ is the electric current of the circuit. $I$ is obtained by measuring the voltage drop $U_{\text {sh }}$ across a shunt resistor of $R_{\mathrm{sh}}=0.1 \Omega$, so that Eq. (10) becomes

$P=U_{\mathrm{gen}} \cdot \frac{U_{\mathrm{sh}}}{R_{\mathrm{sh}}}$.

According to the generator's specifications, the torque $T$ is proportional to the electric current $I$,

$T=k \cdot I$,

with $k=79.9 \mathrm{mN} \mathrm{A}^{-1}$. The turbine features an automatic load control, with the process variable of the controller being the TSR based on hub-height wind speed measurements using a hot-wire probe two-thirds of $D$ upstream of the rotor; see Fig. 2. The generator's load is controlled using an external voltage applied to a field-effect transistor (FET) within the electric circuit; see Schottler et al. (2016) for details. Throughout this study, the TSR was set to $\lambda_{\text {set }}=7$ to ensure a stable point of operation (not in stall) during the experiments.

To measure the thrust force acting on the turbine, it was placed on a three-component force balance (MEMeßsysteme K3D120-50 N). Only the thrust force in main flow direction is considered; thus,

$F=F_{\text {thrust }, x}$.
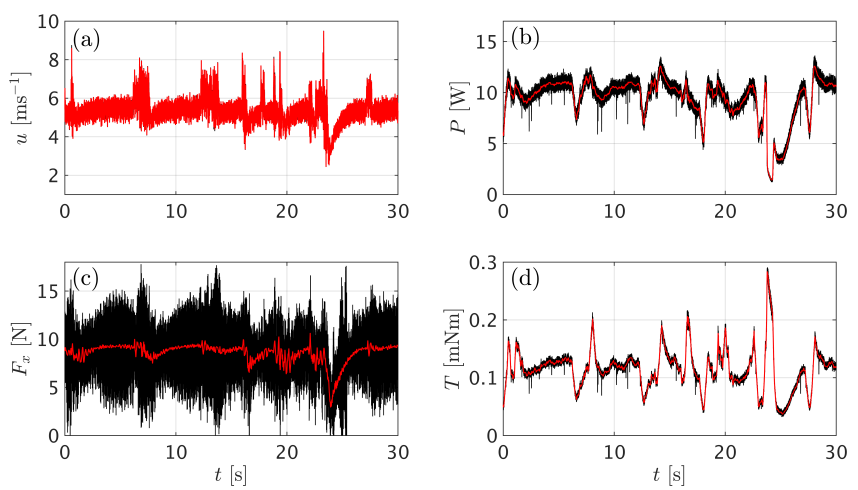

Figure 4. Original (black) and filtered (red) example time series of the wind speed (a), power (b), thrust force (c) and torque (d). The wind speed was filtered using a sixth-order Butterworth low-pass filter at $2 \mathrm{kHz}$. In a similar way, the power and torque signals were filtered at $45 \mathrm{~Hz}$ and the thrust force at $15 \mathrm{~Hz}$.

The setup is sketched in Fig. 2; Fig. 3 shows a photograph. As shown in Fig. 2, three hot wires were installed upstream of the rotor during turbine operation. In contrast to the flow characterization, only the center hot-wire signal at hub height was used when comparing inflow data to turbine data as done in Sect. 4.2.

\subsection{Data processing}

For each experiment, data were recorded simultaneously. During flow characterization the three hot-wire probes were synchronized and during turbine data acquisition the thrust force, power, torque and hot-wire signals were recorded synchronously. Generally, all data sets are superimposed with some kind of measurement noise, which we generally want to exclude from our analyses, while preserving the fluctuations of the turbine signals resulting from the inflow. The data sets are filtered using a sixth-order Butterworth low-pass filter at a cutoff frequency of $15 \mathrm{~Hz}$ for the thrust data and $45 \mathrm{~Hz}$ for the power and torque data. Further details about the approach are shown in Appendix A. Figure 4 shows examples of the time series of the four different signals, filtered and unfiltered. The graph in Fig. 4a shows the wind speed during the intermittent inflow upstream of the turbine. The other graphs show the simultaneously recorded signals of the turbine. Only the filtered data sets are used for further analyses.

\subsection{Choice of scales}

As previously described, we will consider increment PDF of different timescales, $p\left(u_{\tau}\right)$. Defining relevant scales for WECs is not trivial and is the subject of discussion throughout the research community (van Kuik et al., 2016). Therefore, a broad spectrum of timescales were chosen, ranging from the order of seconds to the smallest scale possible while applying the described filtering. By using Taylor's hypothesis 

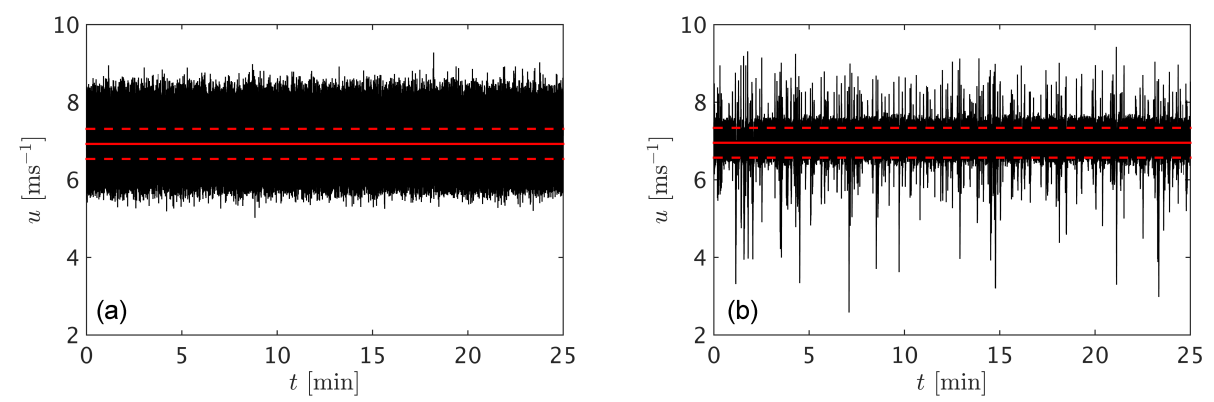

Figure 5. Velocity time series as defined in Eq. (9) of the two inflows considered, A (Gaussian, a) and B (intermittent, b). Further information is shown in Table 3. Solid red lines mark $\langle u(t)\rangle$ and dashed red lines indicate $\langle u(t)\rangle \pm \sigma_{u}$.
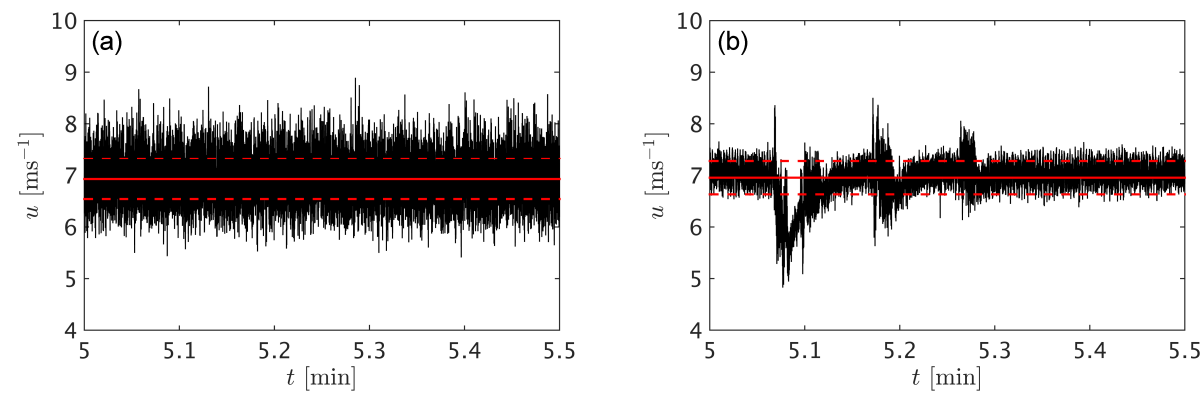

Figure 6. Excerpts of both time series shown in Fig. 5.

of frozen turbulence (Mathieu and Scott, 2000), the chosen timescales are related to length scales of the model turbine, with $\langle u\rangle \approx 7 \mathrm{~m} \mathrm{~s}^{-1}$. The largest scale considered is $\tau=2 \mathrm{~s}$. Thus, the turbine experiences a flow situation corresponding to a $14 \mathrm{~m}$ structure in the wind field impacting the model turbine. Smaller timescales are based on turbine dimensions and the filter frequencies. Table 2 gives an overview of the different scales considered. When analyzing thrust data, the smallest timescale, $\tau=25 \mathrm{~ms}$, was excluded due to the filtering of the data.

\section{Results}

\subsection{Inflow}

Throughout the following analyses, two different flow situations will be considered and used as inflow conditions for the model wind turbine. Figure 5 shows the two wind speed time series as defined in Eq. (9) with $\langle u(t)\rangle \pm \sigma_{u}$ indicated. Additionally, Table 3 lists the mean values, standard deviations and turbulence intensities for the two cases and Fig. 6 shows a $30 \mathrm{~s}$ excerpt. We refer to the time series as inflow A and inflow B, according to Fig. 5. It is noteworthy that in describing the wind fields by their mean values and turbulence intensities, as it is widely done, both conditions A and B are virtually equivalent, as can be seen in Table 3 .
However, just by looking at the time series, a difference becomes obvious, which will be investigated further. Therefore, Fig. 7 shows the increment PDF $p\left(u_{\tau}\right)$ of both time series for the scales listed in Table 2. Clearly, both flows are significantly different regarding intermittency. While inflow A follows a Gaussian trend, inflow B shows a strongly heavytailed, highly intermittent distribution of increments. Therefore, extreme events occur significantly more frequently in inflow B as compared to inflow A. Similar discrepancies as shown in Fig. 1 for offshore measurements and simulated data become obvious. It should be noted that the flows do not aim to reproduce atmospheric wind speed time series. We focus on the statistical properties for chosen timescales as shown in Fig. 7.

\subsection{Turbine reaction}

Next, we investigate the performance data of the model wind turbine when exposed to both A and B flows. To begin with, we consider the thrust force in main flow direction, $p\left(F_{\tau}\right)$, in Fig. 8. Clearly, the difference between Gaussian and nonGaussian inflow conditions remains present in the thrust data for all timescales considered. Non-Gaussian increments are not filtered by the rotor. Going further, we directly compare the normalized quantities, $p\left(F_{\tau}\right)$ and $p\left(u_{\tau}\right)$, separately for both flow conditions in Fig. 9. Neither for the Gaussian nor for the intermittent case can a change in the forms of the increment PDF be observed. Thus, we conclude that the nonGaussian character of the inflow is not averaged out by the 
Table 2. Overview of scales considered in relation to certain characteristic turbine lengths. The timescales $\tau$ were used in the analyses. To get an idea of the spatial dimension, Taylor's hypothesis is used to transfer from time to space with $\langle u\rangle \approx 7 \mathrm{~m} \mathrm{~s}^{-1}$. The obtained length scales are expressed as multiples of the rotor diameter for better comparison. The lengths are further related to physical objects of the turbine to get a sense of the dimensions.

\begin{tabular}{lrrrr}
\hline & Scale 1 & Scale 2 & Scale 3 & Scale 4 \\
\hline Timescale $\tau[\mathrm{s}]$ & 2 & 0.08 & 0.067 & 0.025 \\
Length $/ D[-]$ & $\approx 24$ & 1 & $\approx 0.8$ & 0.3 \\
Physical object & - & rotor diameter & - & order of blade length
\end{tabular}

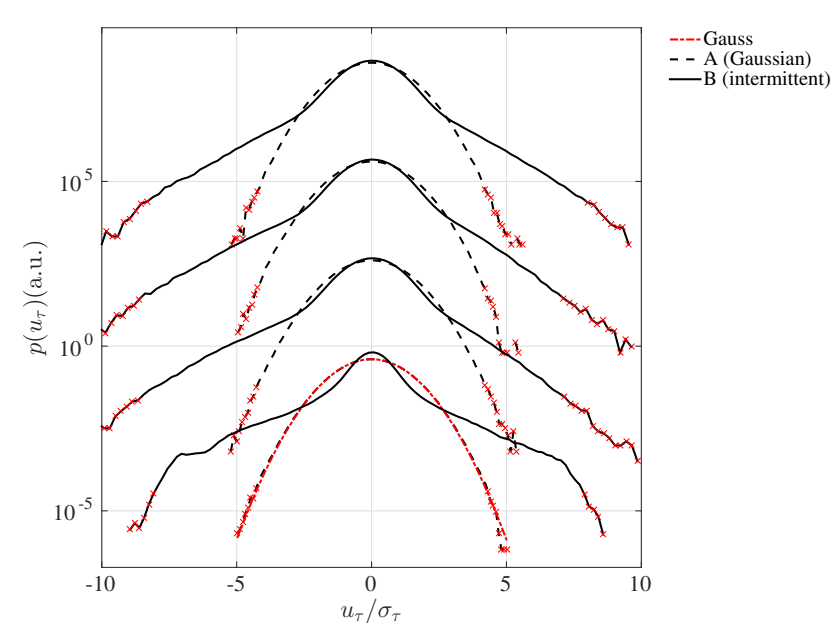

Figure 7. $p\left(u_{\tau}\right)$ of both velocity time series shown in Fig. 5, A (dashed) and B (solid), for $\tau=\{25,67,80 \mathrm{~ms}, 2 \mathrm{~s}\}$ from top to bottom. The different scales are shifted vertically for presentation. A Gaussian fit (dashed red line) of $p\left(u_{\tau=2 \mathrm{~s}}\right)$ for inflow $\mathrm{A}$ is added to guide the eye.

Table 3. First two statistical moments of the time series shown in Fig. 5 and their turbulence intensities. Values are rounded to two decimal places.

\begin{tabular}{lrrr}
\hline Time series & $\langle u(t)\rangle\left[\mathrm{m} \mathrm{s}^{-1}\right]$ & $\sigma_{u}\left[\mathrm{~m} \mathrm{~s}^{-1}\right]$ & TI [\%] \\
\hline A & 6.92 & 0.39 & 5.59 \\
B & 6.96 & 0.38 & 5.50 \\
\hline
\end{tabular}

rotor. In Figs. 8 and 9, the smallest timescale of $\tau=25 \mathrm{~ms}$ is not shown for the thrust data since that scale interferes with the previously applied low-pass filter as described in Sect. 3.2.

So far, we have considered the thrust force of the turbine as an example, showing a transfer of intermittency from $u_{\tau}$ to $F_{\tau}$ by the system dynamics of the turbine. For the power and torque we obtain similar results as for the thrust; thus, we present all quantities for the intermittent inflow together in Fig. 10. None of the quantities smooth out the intermittent inflow to a close-to-Gaussian distribution. Minor deviations of the respective increment PDFs are discussed in Sect. 5.

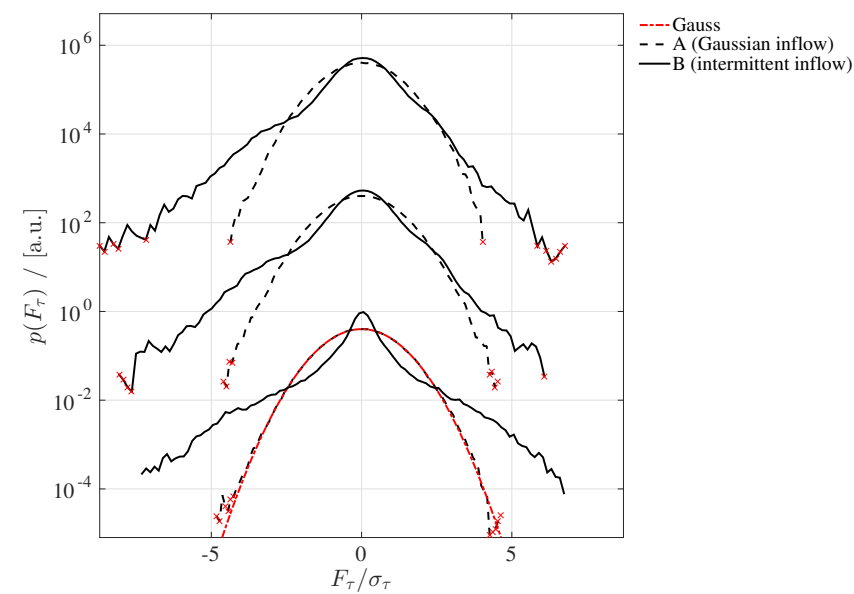

Figure 8. $p\left(F_{\tau}\right)$ of the turbine's thrust force (in main flow direction) exposed to the inflow conditions A (dashed) and B (solid) for $\tau=\{67,80 \mathrm{~ms}, 2 \mathrm{~s}\}$ from top to bottom. The different scales are shifted vertically for presentation.

\section{Discussion}

To what extent non-Gaussian wind statistics impact WECs is an ongoing discussion throughout the wind energy research community. Using an active grid to create different turbulent inflow conditions allows experimental investigations of the impact of turbulence on wind turbines. This study can therefore supplement present approaches in the literature that investigate the impact of non-Gaussianity based on numerical simulations (e.g., Mücke et al., 2011; Gong and Chen, 2014; Berg et al., 2016) or field measurements (Milan et al., 2013). However, when using the model wind turbine to grasp the impact of the different inflows considered, we do not claim full scalability. There is a Reynolds number mismatch between the scaled laboratory model and full-scale turbines. Furthermore, the model is not aero-elastically scaled. Therefore, a detailed study of the (time-) scale dependency of the results is not included here, but chosen timescales as described in Sect. 3.3 are analyzed. The flows described in Sect. 4.1 were generated with a focus on their statistical properties on those scales. The focus is not on details of the time sequences or spectral properties. They aim to reproduce the discrepancy 

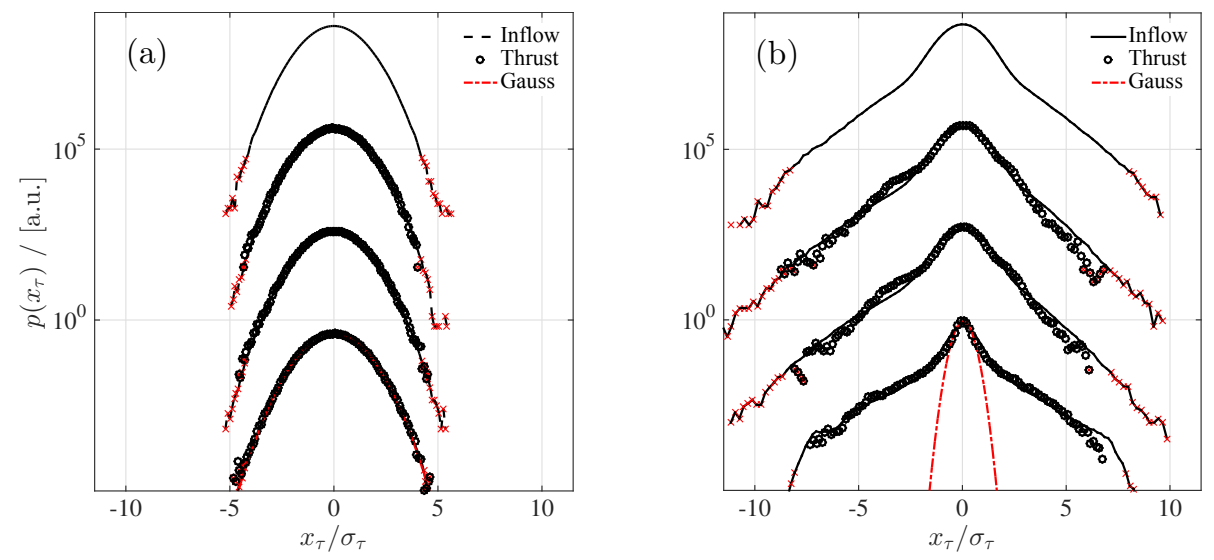

Figure 9. $p\left(u_{\tau}\right)$ (lines) and $p\left(F_{\tau}\right)$ (circles) for both Gaussian (a) and intermittent (b) inflow conditions. Scales as in Fig. 8 from top to bottom, $\tau=\{25,67,80 \mathrm{~ms}, 2 \mathrm{~s}\}$, shifted vertically for presentation.

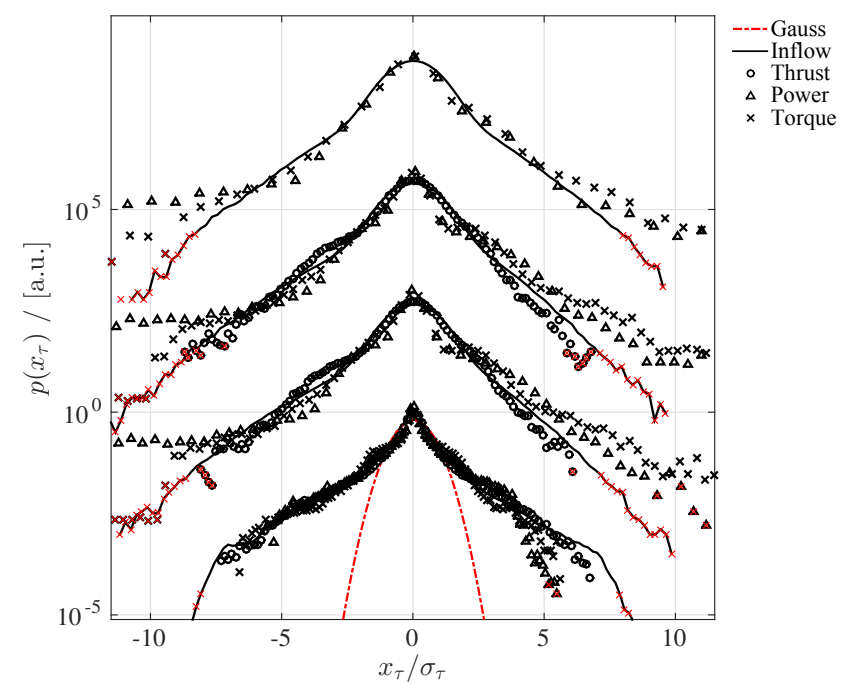

Figure 10. $p\left(x_{\tau}\right)$ for the intermittent inflow condition (line, see Fig. 5a), thrust (circles), power (triangles) and torque (crosses). Scales as in Fig. 8 from top to bottom, $\tau=\{25,67,80 \mathrm{~ms}, 2 \mathrm{~s}\}$, shifted vertically for presentation.

between industry standards and atmospheric wind data in terms of increment statistics.

When processing the experimental data, signal fluctuations not resulting from the inflow are excluded from the analysis by previously applied low-pass filters. While noise is only a minor issue considering the power and torque, the thrust data from the force balance are significantly superimposed by signal fluctuations resulting from the setup itself; see Fig. 4c. These most likely arise from vibrations of the whole setup during turbine operation, ranging from the turbine itself and the support to the fixation in the ground. These fluctuations are of an amplitude that would influence the analysis; however, they are of a higher frequency than the cutoff frequency of the applied low-pass filter. Therefore, they are indeed excluded from the analysis. At the same time, the procedure described in Appendix A might filter fluctuations of higher frequency than the respective cutoff, which are actually directly related to wind speed variations. As a result, minimal timescales have to be set, potentially excluding interesting results for smaller scales. Considering Fig. A2a, the coherence of the hot-wire signal and the thrust data is almost lost completely at approximately $10 \mathrm{~Hz}$. Since this corresponds to a timescale of $\tau=0.1 \mathrm{~s}$ or a length scale of $0.7 \mathrm{~m}$ $(\approx 1.2 \mathrm{D})$, a cutoff frequency of $15 \mathrm{~Hz}$ was chosen in order to include a scale between the rotor diameter and the blade length. From the analysis of other intermittent data, it can be shown that our filtering does not affect the intermittency effects in a significant way. Thus, the filtering only suppresses noise effects.

There might also be aerodynamic effects that are of even higher frequency than the inflow fluctuations and are therefore not captured due to the filtering. Such effects at the rotor are possibly excluded by the low-frequency filtering. This study, however, focuses on dynamics caused by the inflow turbulence.

Considering Fig. 10, some minor deviations between the increment PDF of the inflow and the turbine data can be observed. The torque and the power as defined in Eqs. (12) and (11) are part of the electrical circuit and are therefore directly linked to the manipulative variable of the controller, being the voltage applied to the FET, $U_{\mathrm{FET}}$. Thus, an analysis of those quantities includes not only fluctuations caused by the inflow but also those resulting from the controller. As overshoots are typical for closed-loop control systems (Ogunnaike and Ray, 1994; Chien and Chung, 2003), they likely bias the present analysis, especially for small timescales regarding the power and the torque. This most likely causes the asymmetric distributions of power and torque increments in Fig. 10. Because of this, the focus of the analysis is on the thrust data. Nonetheless, the main finding that all quantities feature strongly intermittent distributions of increments re- 
mains, despite differences among the parameters, as Fig. 10 shows.

\section{Conclusions}

In this study, an experimental setup that allows the investigation of interactions between various turbulent flows with a model wind turbine was realized. Experiments were performed in order to elaborate on the impact of non-Gaussian wind statistics on WECs. Our results do not show any filtering of the intermittent features of wind fields found in the atmosphere by the turbine. Consequently, one should be aware that wind characteristics, which are not reflected in standard wind field descriptions, the IEC 61400-1 for example, have a significant impact on wind turbines. Intermittent inflow is converted to similarly intermittent turbine data on all scales considered, ranging down to sub-rotor scales. Thus, statistical properties of the inflow time series that are not captured by describing them using one-point statistics are of relevance and should be included in standards characterizing inflow conditions. If intermittent inflows lead to intermittent loading, including extreme loads that occur much more frequently than currently modeled in the standards, then this has implications for the use of the current standards in designing wind turbines to withstand the wind conditions experienced.

\section{Data availability}

The offshore data analyzed in this paper was made available by the DEWI and the Federal Maritime and Hydrographic Agency (German Bundesamt für Seeschifffahrt und Hydrographie). Access can be requested through http://fino.bsh.de/ (last access: 6 January 2017).

Furthermore, the experimental datasets are archived by the University of Oldenburg. The data can be made available by contacting the corresponding author. 
Appendix A: Data processing - coherence analysis

As described in Sect. 3.2, the raw data sets are superimposed by measurement noise, which should be excluded from the analyses. Since we analyze different parameters, an appropriate filtering of the different raw signals should, nonetheless, allow a comparison of their statistics. This section shows the procedure of choosing appropriate filter frequencies. To begin with, $u(t)$ during the intermittent inflow $\mathrm{B}$ is filtered using a sixth-order Butterworth low-pass filter. The cutoff frequency is set to $2 \mathrm{kHz}$ since high-frequency noise, which is typical for hot-wire anemometers (Jørgensen and Hammer, 1999), should be filtered. Furthermore, the resolved length scales corresponding to $2 \mathrm{kHz}$ ( $\sim \mathrm{mm}$, using Taylor's hypothesis; Mathieu and Scott, 2000) are reasonably small for our purposes. Figure A1 shows the PSD of both flows based on raw and filtered data. Since we want to concentrate on the fluctuations of turbine data caused by the inflow, we estimate a maximal frequency for which the fluctuations of the respective turbine data are coherent with the fluctuations of the filtered velocity signal. Therefore, we consider the magnitudesquared coherence, $\gamma_{u^{\prime} x^{\prime}}^{2}=\frac{\left|P_{u^{\prime} x^{\prime}}(f)\right|^{2}}{P_{u^{\prime} u^{\prime}}(f) P_{x^{\prime} x^{\prime}}(f)}$,

of the filtered wind speed fluctuations of the intermittent inflow (B) and the fluctuations of the respective turbine quantity $x^{\prime}$ (Carter et al., 1973), with $x$ being the power, torque or thrust. $P_{u^{\prime} x^{\prime}}$ denotes the cross-spectral density; $P_{u^{\prime} u^{\prime}}$ and $P_{x^{\prime} x^{\prime}}$ denote the autospectra. The results are shown in Fig. A2. At the values indicated by the dashed red lines in Fig. A2, the coherence of the signals is almost completely lost. Therefore, we choose a cutoff frequency of $15 \mathrm{~Hz}$ for the thrust data and $45 \mathrm{~Hz}$ for the power and torque data to filter the raw data using a sixth-order Butterworth low-pass filter. Hereby, higher frequencies are excluded, as only fluctuations resulting from the inflow should be considered.

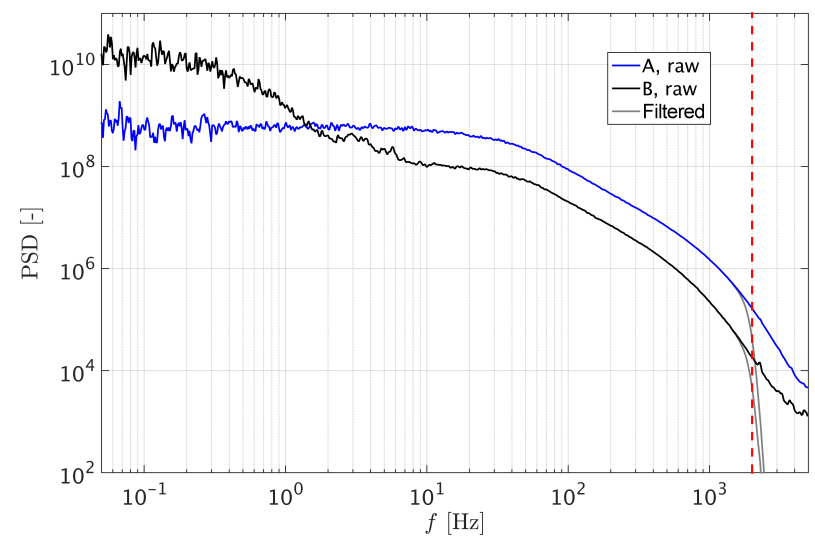

Figure A1. Power spectral density (PSD) of $u(t)$ for the Gaussian inflow A (blue) and the intermittent inflow B (black). The effect of a sixth-order Butterworth low-pass filter at $f_{\text {cut }}=2 \mathrm{kHz}$ is shown in gray. The dashed red line marks $f_{\text {cut }}=2 \mathrm{kHz}$.
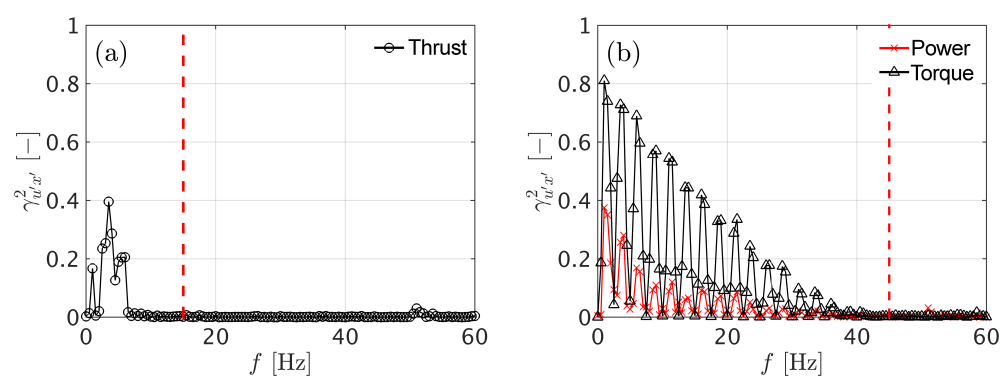

Figure A2. Magnitude-squared coherence of filtered hot-wire data and thrust (a) as well as power and torque (b). Used here were 500 Hanning windows with $50 \%$ overlap, as suggested by Carter et al. (1973). Panel (b) shows regular drops of $\gamma^{2}$, which are caused by a filter function within the control algorithm of the model turbine. Since the controller affects the electrical circuit, there is a direct connection to the electrical current and therewith to the power and torque. Consequently, the effect of the filter is clearly visible in this graph. 


\section{Appendix B: Variances of increment PDF}

For completeness, the variances $\sigma_{\tau}^{2}$ of every time series of increments, $x_{\tau}$, are shown in Table B1 for the synthetic and offshore data (see Fig. 1) and for the experimental data in Table B2.

Table B1. Variances of each increment time series, $u_{\tau}(t)$, for synthetic data based on the Kaimal model and field data.

\begin{tabular}{lrrrrr}
\hline Timescale $\tau[\mathrm{s}]$ & 1 & 5 & 10 & 30 & 60 \\
\hline $\operatorname{var}\left(u_{\tau}\right)$, Kaimal $\left[\mathrm{m}^{2} \mathrm{~s}^{-2}\right]$ & 0.25 & 0.47 & 0.53 & 0.58 & 0.58 \\
$\operatorname{var}\left(u_{\tau}\right)$, FINO1 $\left[\mathrm{m}^{2} \mathrm{~s}^{-2}\right]$ & 0.04 & 0.11 & 0.15 & 0.24 & 0.31 \\
\hline
\end{tabular}

Table B2. Variances of each increment time series for the experimental data. $\operatorname{var}\left(u_{\tau}\right)$ corresponds to the graphs shown in Fig. 7, var $\left(F_{\tau}\right)$ to the graphs in Fig. 8, $\operatorname{var}\left(P_{\tau}\right)$ and $\operatorname{var}\left(T_{\tau}\right)$ to $p\left(P_{\tau}\right)$ and $p\left(T_{\tau}\right)$, respectively, as shown in Fig. 10.

\begin{tabular}{lrrrr}
\hline Timescale $\tau[\mathrm{s}]$ & 0.025 & 0.067 & 0.08 & 2 \\
\hline $\operatorname{var}\left(u_{\tau}\right)$, Inflow A $\left[\mathrm{m}^{2} \mathrm{~s}^{-2}\right]$ & 0.30 & 0.30 & 0.30 & 0.30 \\
$\operatorname{var}\left(u_{\tau}\right)$, Inflow B $\left[\mathrm{m}^{2} \mathrm{~s}^{-2}\right]$ & 0.06 & 0.08 & 0.09 & 0.252 \\
\hline $\operatorname{var}\left(F_{\tau}\right)$, Inflow A $\left[\mathrm{m}^{2} \mathrm{~s}^{-2}\right]$ & - & 0.03 & 0.06 & 0.13 \\
$\operatorname{var}\left(F_{\tau}\right)$, Inflow B $\left[\mathrm{m}^{2} \mathrm{~s}^{-2}\right]$ & - & 0.11 & 0.14 & 0.86 \\
\hline $\operatorname{var}\left(P_{\tau}\right)$, Inflow A $\left[\mathrm{m}^{2} \mathrm{~s}^{-2}\right]$ & 0.03 & 0.12 & 0.16 & 3.31 \\
$\operatorname{var}\left(T_{\tau}\right)^{*}$, Inflow B $\left[\mathrm{m}^{2} \mathrm{~s}^{-2}\right]$ & 1.17 & 6.28 & 8.48 & 133.72 \\
\hline
\end{tabular}

$* \times 10^{-5}$. 
Acknowledgements. We thank the Reiner Lemoine Stiftung (RLS) for funding parts of this work and Stefan Ivanell for providing the rotor blade design. Furthermore, we thank Philip Rinn and Matthias Wächter for fruitful discussions as well as the German Bundesamt für Seeschifffahrt und Hydrographie (Federal Maritime and Hydrographic Agency) and the DEWI for providing the FINO1 data.

Edited by: H. Hangan

Reviewed by: two anonymous referees

\section{References}

Berg, J., Natarajan, A., Mann, J., and Patton, E. G.: Gaussian vs non-Gaussian turbulence: impact on wind turbine loads, Wind Energy, 17, 1975-1989, doi:10.1002/we.1963, 2016.

Boettcher, F., Renner, C., Waldl, H. P., and Peinke, J.: On the statistics of wind gusts, Bound.-Lay. Meteorol., 108, 163-173, doi:10.1023/A:1023009722736, 2003.

Burton, T., Sharpe, D., Jenkins, N., and Bossanyi, E.: Wind Energy Handbook, John Wiley \& Sons, Ltd, UK, 2001.

Carrasco, J. M., Franquelo, L. G., Bialasiewicz, J. T., Member, S., Galván, E., Guisado, R. C. P., Member, S., Ángeles, M., Prats, M., León, J. I., and Moreno-alfonso, N.: PowerElectronic Systems for the Grid Integration of Renewable Energy Sources: A Survey, IEEE T. Ind. Electron., 53, 1002-1016, doi:10.1109/TIE.2006.878356, 2006.

Carter, G. C., Knapp, C. H., and Nuttall, A. H.: Estimation of the Magnitude-Squared Coherence Function Via Overlapped Fast Fourier Transform Processing, IEEE T. Acoust. Speech., 21, 337-344, doi:10.1109/TAU.1973.1162496, 1973.

Chen, Z. and Spooner, E.: Grid power quality with variable speed wind turbines, IEEE T. Energy Conver., 16, 148-154, doi:10.1109/60.921466, 2001.

Chien, I.-L. and Chung, Y.: Simple PID controller tuning method for processes with inverse response plus dead time or large overshoot response plus dead time, Ind. Eng. Chem. Res., 42, 4461-4477, doi:10.1021/ie020726z, 2003.

De Gaetano, P., Repetto, M. P., Repetto, T., and Solari, G.: Separation and classification of extreme wind events from anemometric records, J. Wind Eng. Ind. Aerod., 126, 132-143, doi:10.1016/j.jweia.2014.01.006, 2014.

Feng, Y., Qiu, Y., Crabtree, C. J., Long, H., and Tavner, P. J.: Monitoring wind turbine gearboxes, Wind Energy, 16, 728-740, doi:10.1002/we.1521, 2013.

Frisch, U.: Turbulence: the legacy of A.N. Kolmogorov, Vol. 1, Cambridge University Press, doi:10.1017/S0022112096210791, 1995.

Gong, K. and Chen, X.: Influence of non-Gaussian wind characteristics on wind turbine extreme response, Eng. Struct., 59, 727744, doi:10.1016/j.engstruct.2013.11.029, 2014.

International Electrotechnical Commission: IEC 61400-1: Wind turbines part 1: Design requirements, International Electrotechnical Commission: IEC 61400-1: Wind Turbines part 1: Design requirements (3rd Edn.), IEC, Geneva, Switzerland, 2005.

Jonkman, B. J.: TurbSim user's guide: Version 1.50, TurbSim User's Guide: Version 1.50, National Renewable Energy Laboratory, Golden, Colorado, USA, 2009.
Jonkman, J. M. and Buhl Jr., M. L.: FAST user's guide, National Renewable Energy Laboratory, Golden, CO, Technical Report No. NREL/EL-500-38230, 2005.

Jørgensen, F. E. and Hammer, M.: Hot-wire anemometry behaviour at very high frequencies, Meas. Sci. Technol., 8, 221-221, doi:10.1088/0957-0233/8/3/002, 1999.

Kaimal, J. C. J., Wyngaard, J. C. J., Izumi, Y., Coté, O. R., and Cote, O. R.: Spectral Characteristics of Surface-Layer Turbulence, Q. J. Roy. Meteor. Soc., 98, 563-589, doi:10.1002/qj.49709841707, 1972.

Larsen, T. J. and Hansen, A. M.: How 2 HAWC2, the user's manual, Ris $\varnothing$ National Laboratory, 2007.

Liu, L., Hu, F., Cheng, X.-L., and Song, L.-L.: Probability Density Functions of Velocity Increments in the Atmospheric Boundary Layer, Bound.-Lay. Meteorol., 134, 243-255, doi:10.1007/s10546-009-9441-z, 2010.

Mathieu, J. and Scott, J.: An introduction to turbulent flow, Cambridge University Press, 2000.

Milan, P., Wächter, M., and Peinke, J.: Turbulent Character of Wind Energy, Phys. Rev. Lett., 110, 138701, doi:10.1103/PhysRevLett.110.138701, 2013.

Morales, A., Wächter, M., and Peinke, J.: Characterization of wind turbulence by higher-order statistics, Wind Energy, 15, 391-406, doi:10.1002/we.478, 2012.

Moriarty, P. J. and Hansen, A. C.: AeroDyn Theory Manual, NREL/EL-500-36881, National Renewable Energy Laboratory, Golden, Colorado, USA, 2005.

Mücke, T., Kleinhans, D., and Peinke, J.: Atmospheric turbulence and its influence on the alternating loads on wind turbines, Wind Energy, 14, 301-316, doi:10.1002/we.422, 2011.

Musial, W., Butterfield, S., and McNiff, B.: Improving Wind Turbine Gearbox Reliability Preprint, European Wind Energy Conference, 1-13, Milan, Italy, 2007.

Nielsen, M., Larsen, G., and Hansen, K.: Simulation of inhomogeneous, non-stationary and non-Gaussian turbulent winds, Journal of Physics: Conference Series, 75, 12060, doi:10.1088/17426596/75/1/012060, 2007.

Odemark, Y.: Wakes behind wind turbines-Studies on tip vortex evolution and stability, Technical Report, Royal Institute of Technology KTH Mechanics, Stockholm, Sweden, 2012.

Ogunnaike, B. A. and Ray, W. H.: Process dynamics, modeling, and control, Vol. 1, Oxford University Press, New York, 1994.

Press, W. H., Teukolsky, S. A., Vetterling, W. T., and Flannery, B. P.: Numerical Recipes in C (2nd Edn.): The Art of Scientific Computing, Cambridge University Press, New York, NY, USA, 1992.

Schlipf, D., Schlipf, D. J., and Kühn, M.: Nonlinear model predictive control of wind turbines using LIDAR, Wind Energy, 16, 1107-1129, doi:10.1002/we.1533, 2013.

Schottler, J., Hölling, A., Peinke, J., and Hölling, M.: Design and implementation of a controllable model wind turbine for experimental studies, Journal of Physics: Conference Series, 753, 072030, doi:10.1088/1742-6596/753/7/072030, 2016.

Sørensen, P., Cutululis, N. A., Vigueras-Rodriguez, A., Jensen, L. E., Hjerrild, J., Donovan, M. H., and Madsen, H.: Power Fluctuations From Large Wind Farms, IEEE T. Power Syst., 22, 958965, doi:10.1109/TPWRS.2007.901615, 2007.

van Kuik, G. A. M., Peinke, J., Nijssen, R., Lekou, D., Mann, J., Sørensen, J. N., Ferreira, C., van Wingerden, J. W., Schlipf, D., Gebraad, P., Polinder, H., Abrahamsen, A., van Bussel, G. J. W., 
Sørensen, J. D., Tavner, P., Bottasso, C. L., Muskulus, M., Matha, D., Lindeboom, H. J., Degraer, S., Kramer, O., Lehnhoff, S., Sonnenschein, M., Sørensen, P. E., Künneke, R. W., Morthorst, P. E., and Skytte, K.: Long-term research challenges in wind energy a research agenda by the European Academy of Wind Energy, Wind Energ. Sci., 1, 1-39, doi:10.5194/wes-1-1-2016, 2016.

Wächter, M., Heißelmann, H., Hölling, M., Morales, A., Milan, P., Mücke, T., Peinke, J., Reinke, N., and Rinn, P.: The turbulent nature of the atmospheric boundary layer and its impact on the wind energy conversion process, J. Turbul., 13, N26, doi:10.1080/14685248.2012.696118, 2012.
Weitemeyer, S., Reinke, N., Peinke, J., and Hölling, M.: Multiscale generation of turbulence with fractal grids and an active grid, Fluid Dyn. Res., 45, 061407, doi:10.1088/01695983/45/6/061407, 2013.

Westerhellweg, A., Neumann, T., and Riedel, V.: Fino1 mast correction, Dewi Magazin, 40, 60-66, 2012. 